\title{
Systemische, soziale und emotionale Herausforderungen und Glücksmomente im beruflichen Alltag von haupt- und nebenberuflichen YouTuber*innen
}

\author{
„Have fun and work at the same time“
}

\author{
Milena Albiez ${ }^{1}$ \\ Angenommen: 30. September 2020 / Online publiziert: 21. Oktober 2020 \\ (c) Der/die Autor(en) 2020
}

\section{Zusammenfassung}

Ziel der vorliegenden Untersuchung in der Zeitschrift Gruppe. Interaktion. Organisation (GIO) war es, die Herausforderungen und Motivationen von YouTuber*innen in ihrer Tätigkeit als Content-Creator herauszufinden und mögliche Ursachen zu benennen. Es konnte anhand von leitfadengestützten Interviews und deren Auswertung dargelegt werden, dass YouTuberinnen und YouTuber in ihrer Tätigkeit als Content Creator auf YouTube mit einem hohen Maß an systemischen, emotionalen und sozialen Belastungen konfrontiert sind. Diese Belastungen führen unter bestimmten Umständen zu einem Erschöpfungszustand oder zum Burnout. Diesen prekären Arbeitssituationen stehen übermäßig positiv beschriebene Glücksmomente gegenüber, die dazu beitragen, dass YouTuber*innen die Tätigkeit weiter fortführen. Die Ursachen dieser Herausforderungen liegen in der Selbstreferenz der Tätigkeit, der Vereinzelung in der Tätigkeit und im Alleinsein in der Tätigkeit, die diese Herausforderungen, als individualisierte Probleme darstellen. Es handelt sich also weniger um individuelle Belastungen, sondern um medientechnologische Umbrüche, sozioökonomischen Veränderungen und Subjektivierung der Arbeitswelt, die dazu geführt haben, dass der Faktor Gruppe in dieser Art von Tätigkeit, zwischen Individuum und Organisation, weniger vorhanden ist.

Schlüsselwörter Social Media · Coaching · Organisation · YouTube · Digitalisierung · YouTuber · Content Creator · Hobby · Selbstständigkeit · Google · Burnout · Stress · Subjektivierung · New Work · Gruppendynamik

Milena Albiez, M.F.A.

hallo@milenaalbiez.de, albiez@uni-kassel.de

1 Universität Kassel, Kassel, Deutschland 


\title{
Systemic, social and emotional challenges and magic moments in the professional life of full-time and part-time YouTubers
}

"Have fun and work at the same time"

\begin{abstract}
Aim of the present article in the journal Gruppe. Interaktion. Organisation (GIO) is to identify the challenges and motivation of YouTubers in their role as content creators and to identify possible causes of these challenges. I demonstrate by means of guided interviews and their evaluation that YouTube creators are confronted with a high degree of systemic, emotional and social stress in their work on content creation. Under certain circumstances, this stress leads to exhaustion or burnout. The precarious work situation is contrasted by positively described moments of happiness, which lead YouTubers to continue with their work. The causes of these challenges can be identified as the self-reference of the activity, the isolation and loneliness within the activity. These challenges are reflected as individualized problems. It is less about individual burdens, than rather about media-technological upheavals, socio-economic changes and subjectification of working environments, which have led to the phenomenon that the group as a determining factor is less present in this work, between the individual and the organization.
\end{abstract}

Keywords Social media - Coaching · Organization - YouTube - Digitalization · YouTuber · Content creator · Hobby · Self-reliance $\cdot$ Google $\cdot$ Burnout $\cdot$ Stress $\cdot$ Subjectification $\cdot$ New work $\cdot$ Group dynamics

\section{Einleitung}

YouTube ist mit mehr als zwei Milliarden Nutzern'1, die erfolgreichste Plattform für Videocontent im Internet. Von Wissensvermittlung bis Unterhaltung werden pro Tag laut Pressemitteilug von YouTube insgesamt eine Milliarde Stunden Wiedergabezeit angesehen. Neben Videos von z. B. Musik-Labels und Fernsehsendern gibt es im Verhältnis dazu eine kleinere Anzahl von Videos, in denen YouTuber*innen (Content Creator) eigene Videos zu unterschiedlichen Themen konzipieren, produzieren und hochladen ${ }^{2}$. Die Tätigkeit von Video- und Bildproduzent*innen wurde weniger beforscht. Mitte Mai 2018 berichtete die damals 19-jährige US-amerikanische YouTuberin Elle Mills ${ }^{3}$, mit ca. 1,5 Mio. Abonnenten (geschätztes Monatseinkommen zwischen 510 und $8700 €^{4}$ ), in einem Video über ihren Burnout. „This is all I ever wanted and why the fuck am I so unfucking unhappy? It doesn't make any fucking sense! [...] Because, like this is literally my fucking dream and I am fucking so un-fucking-happy! ". Dieses und andere Videos von YouTuber*innen weltweit lösten ein breites

\footnotetext{
${ }^{1}$ Eigene Angaben von YouTube: <https://www.youtube.com/yt/about/ press/> [16.05.2020].

2 Über den Einfluss von Social Media und Videoplattformen auf ihre Rezipienten gibt es mittlerweile eine breite Diskussion in Studien und Fachartikeln z.B. aus den Bereichen Psychologie, Medienpädagogik, Kunst und Design oder Kulturwissenschaften.

${ }^{3}$ Elle Mills, „ElleOfTheMills“: „Burnt Out At 19“ vom 18.05.2018 $<$ https://www.youtube.com/watch?v=WKKwgq9LRgA> [16.05.2020]

$4<$ www.socialblade.com> [16.05.2020].
}

Medienecho ${ }^{5}$ aus. Was sind die Gründe dafür, dass sehr junge Menschen in diesem Bereich, der für Unterhaltung bekannt ist, über Erschöpfungszustände berichten? Nun mag sich die Leserschaft vielleicht fragen, weshalb man Videos für YouTube produziert und was es für einen Sinn ergibt, den eigenen Tag mit der Kamera festzuhalten, die eigene Meinung zu Themen kundzutun, den Zuschauern den Einkauf zu zeigen oder Mentos-Bonbons in Cola-Flaschen $\mathrm{zu}$ stecken. Jedoch ist YouTuberIn heute ein Traumberuf für Kinder und Jugendliche, auch wenn es nur wenige Personen schaffen mit ihrem Content auf Dauer erfolgreich zu sein und ein regelmäßiges Einkommen zu haben (Meyer 2019). Wie im Wortstamm des Wortes „Creator“ ersichtlich wird, ,erschaffen“ oder „kreieren“ YouTuber*innen auf ihren Kanälen täglich bis wöchentlich neues Videomaterial. „The reason I wanted to do YouTube, is because I think it's one of the ultimate ways to have fun and work at the same time. You get to completely express yourself and make things constantly. That's fucking insanely cool. " ${ }^{\circ}$, nennt der YouTuber Bobby Burns als seine größte Motivation, trotz seines Burnouts weiter auf YouTube aktiv zu sein. Die diesem Artikel zugrunde liegende qualitativ empirische Studie hatte zum Ziel, die Diskrepanz zwischen hoher Motivation, vielbesprochenen Magic Moments und Herausforderungen in der Tätigkeit von YouTuber*innen zu untersuchen und Zusammenhänge für mögliche Ursachen herzustellen.

\footnotetext{
5 vgl. The Guardian online vom 08.09.2018: „The YouTube stars heading for burnout: ,The most fun job imaginable became deeply bleak“" <https://www.theguardian.com/technology/2018/sep/08/ youtube-stars-burnout-fun-bleak-stressed $>$ [16.05.2020].

${ }^{6}$ Burns, Bobby: „I Had A Breakdown (Mental Health On YouTube)“ vom 04.05.2018<https://www.youtube.com/watch?v=142yMJpC32o> [16.05.2020].
} 


\section{Kontext YouTube}

YouTube wurde 2005 von Chad Hurley, Steve Chen und Jawed Karim gegründet. Der eigentliche Zweck der Plattform war es, Videos möglichst unkompliziert, zwischen Freunden und Familie, im Internet zu teilen. Außerdem war es so möglich sich über die Plattform mit anderen Nutzern $\mathrm{zu}$ verbinden und die Videos via HTML-Code auf anderen Webseiten einzubetten (Burgess und Green 2018). Der Slogan „Broadcast yourself“, welcher bis 2010 in das Logo von YouTube integriert war zeigt, dass sich das Konzept von einem privaten Depot für selbstgefilmte Videos zu einer Plattform auf welcher man ,sich selbst sendet“ gewandelt hat. Die YouTube LLC ist eine Tochtergesellschaft der Google LLC, welche seit 2015 Teil der Alphabet Inc. Holding ist und umfasst neben der bekannten Suchmaschine, andere Unternehmen mit dem Fokus auf Künstliche Intelligenz, Biotechnologie oder autonomes Fahren.

\subsection{Mit YouTube Geld verdienen}

Um auf YouTube Geld zu verdienen, muss man zunächst in das YouTube Partnerprogramm aufgenommen werden. Um die Aufnahme müssen sich Interessenten bewerben und folgende Voraussetzungen erfüllen: Richtlinien des YouTubePartnerprogramm einhalten, Wiedergabezeit von mehr als $4000 \mathrm{~h}$ im letzten Jahr, mehr als 1000 Abonnenten etc. ${ }^{7}$ Um YouTube-Partner zu werden, muss das Video-Material vorwiegend von der Person stammen, die YouTube-Partner werden will. Über den Verdienst darf laut den Richtlinien des Partnerprogramms nicht gesprochen werden. YouTuber*innen werden von YouTube an Werbeeinnahmen beteiligt, in dem neben, in oder nach dem Video Werbeinhalte gezeigt werden ${ }^{8}$. Daneben gibt es noch die Möglichkeit mit Marken oder Organisationen zusammenzuarbeiten und z. B. für Produktplatzierungen entlohnt zu werden. Kanäle mit weniger Abonnenten erhalten oft Produkte kostenlos und drehen dann darüber ein Video, was als Sponsoring bezeichnet wird. Die Einnahmen aus sogenannten „Kooperationen“ im Influencer-Marketing (Jahnke 2018) bilden heute einen Großteil des Einkommens von YouTuber*innen ab 100.000 Abonnenten, da der Erlös aus der Werbeeinnahmen allein, für die Deckung der Lebenshaltungskosten nicht ausreicht.

\footnotetext{
$7<$ https://support.google.com/youtube/answer/72851\#reviewprocess> [16.05.2020].

$8<$ https://support.google.com/youtube/answer/2467968> [16.05.2020].
}

\subsection{Einfluss von Algorithmen}

Die Funktions- und Wirkungsweise von YouTube-Algorithmen sind im Gegensatz zu anderen Open-Source'-Plattformen nicht für die Allgemeinheit einsehbar, die ungefähren Funktionsweisen sind dem Fachpublikum jedoch bekannt. Die Plattform arbeitet mit Algorithmen, die Videos in ein Ranking einsortieren (Marek 2013). Hier findet eine Zuordnung von Relevanz statt, da Videos unterschiedlich viel Beachtung erfahren, je nachdem wie viele Zuschauer das Video wie lange ansehen und interagieren (Kommentar, Like, Dislike) (Rieder et al. 2018). Neben den Aufrufzahlen ist mittlerweile die Watch-Time, also die Zeit, die User das Video tatsächlich ansehen, wichtig. So kann ermittelt werden, ob das Video mit dem Thumbnail ${ }^{10}$ und dem Titel, den Erwartungshaltungen der Zuschauer*innen entsprochen hat. 2016 wurden vor allem Suchalgorithmen auf YouTube umstrukturiert. Seitdem steht das Deep Learning, also das maschinelle Lernen der Algorithmen, beim Suchen von Zuschauern für bestimmten Content im Vordergrund. Die Watch History, Search History und andere demografische Informationen der Zuschauer*innen werden gesammelt und analysiert. Das heißt, dass vor allem YouTuber*innen, deren Videos vermutlich niedrigere Rankings haben, da sie von weniger Personen abonniert sind, mehr Videos produzieren müssen, wenn sie gerne mehr Abonnenten oder Aufrufzahlen haben wollen. Die Regelmäßigkeit beim Upload eines Videos soll das Verbleiben an einer Stelle im Ranking oder langsames Aufsteigen im Ranking garantieren. Nach Änderungen in den Funktionsweisen der Algorithmen, wurden von YouTuber*innen deutlich längere Videos hochgeladen, die mit dem entsprechend größeren Produktionsaufwand verbunden sind.

\subsection{YouTuber*innen}

Der Begriff YouTuber wird im deutschsprachigen Raum zur Beschreibung einer Person (Burgess und Green 2009) genutzt, die einen eigenen Kanal hat und Videos produziert. Als gängiges Äquivalent dazu wird im englischen Sprachraum der Begriff YouTube Creator genutzt. Dies zeigt sich z.B. in der Betrachtung der deutschen ${ }^{11}$ und englischen ${ }^{12}$ Version der YouTube Partnerprogramm-Seite. Daneben

\footnotetext{
9 Open Source wird als Bezeichnung für Software genutzt, deren Quelltext und Programmierung öffentlich ist. Sie kann von allen eingesehen, genutzt und geändert werden und ist meistens für die User kostenlos.

10 Thumbnail: dt. Vorschaubild, welches zuerst vor dem Start eines Videos angezeigt wird. Dies kann ein Videostill oder ein bearbeitetes Bild sein.

$11<$ https://www.youtube.com/intl/de/creators/benefits/> [16.05.2020].

$12<$ https://www.youtube.com/intl/eng/creators/benefits/> [16.05. 2020].
} 
existiert der Begriff YouTube-Star oder YouTube-celebrity (Lange 2007), der Personen beschreibt, die innerhalb und außerhalb von YouTube einen bestimmten Bekanntheitsgrad erlangt haben. Innerhalb von YouTube und auch während der Interviews werden und wurden die Adjektive „groß“ und „klein“ genutzt, um die Kanalgröße, also die Abonnentenzahl zu beschreiben. Diese Beschreibung wird zum einen in Fachliteratur im Marketingbereich (Jahnke 2018) genutzt, zum anderen werden auf der YouTubeSeite $^{13}$ YouTuber*innen in bestimmte Größenkategorien eingeteilt, die mit zunehmender Abonnentenzahl mehr Vorteile haben. Der deutschsprachige YouTube-Standort in Deutschland, Österreich und der Schweiz ist für deutschsprachige-YouTuber*innen aufgrund der demografischen Faktoren und potenziellen Zielgruppen limitiert. Englischsprachige YouTuber*innen erreichen international größere Zielgruppen (Opresnik und Yilmaz 2016). Als YouTuber*in nutzt die Person YouTube genauso, wie andere YouTubeNutzer, die keinen eigenen Kanal betreiben, ,weshalb eine strikte Trennung zwischen Produzenten und Rezipienten nicht mehr sinnvoll erscheint. Bei den Videoplattformen im Internet ist jeder Empfänger auch ein potenzieller Sender.“ (Marek 2013, S. 45).

\subsection{YouTube als Beruf}

Um haupt- oder nebenberufliche YouTuber*in zu werden, bedarf es vermeintlich keiner Berufsausbildung. Jeder kann ein Google-Konto und somit einen Kanal anlegen. Dieser niedrigschwellige und kostenlose Zugang hat den Anreiz, es einfach einmal auszuprobieren. Ob ein Kanal „erfolgreich" ist oder nicht, hängt jedoch von vielen Faktoren ab, die meist während der ersten Tätigkeiten auf YouTube erlernt werden. Learning by doing ist neben Tutorials auf der Plattform selbst oder Publikationen und Handbüchern, die im Handel erhältlich sind, einer der wichtigsten Faktoren in Karrierewegen von YouTuber*innen. Einige der deutschen YouTuber*innen haben ihre Kanäle als Hobby ${ }^{14}$ begonnen und konnten diese später als ihre Haupterwerbsquelle angeben. Diese Entwicklung vom Hobby zum Beruf, ist eine Besonderheit. Es gibt keine klassische Berufsausbildung und die Tätigkeit ist im Allgemeinen auch in seinen Tätigkeitsfeldern umfassender, als der Ausbildungsberuf Mediengestalter*in Bild und Ton. YouTube und Content Creation im Allgemeinen ist laut Experten ein zukunftsfähiges Berufsfeld (Lohmaier und Schäfer 2016). Da sich vor einigen Jahren gezeigt hat, dass es möglich ist auf YouTube Geld zu verdienen und sich die Werbeindustrie auf sogenanntes In-

\footnotetext{
13 < https://www.youtube.com/intl/de/creators/benefits/> [16.05.2020].

14 Barbara Sofie: „YouTube als Job - Was ihr schon immer wissen wolltet" vom 25.04.2018<https://www.youtube.com/watch? $\mathrm{v}=$ So7A7g09Jb8 $>$ [16.05.2020].
}

fluencermarketing (vgl. Jahnke 2018) ausweitet, haben sich Motive auch dahingehend verändert, dass ein YouTube-Kanal aufgrund des möglichen Verdiensts gestartet wird. In einer Studie von Duffy und Wissinger (2017) wurden Social-Media-Karrieren hinsichtlich kreativer Arbeit in digitalen Ökonomien untersucht. In der Studie wird deutlich, dass der Mythos der spaßigen, kreativen (Social-Media) Arbeit, Herausforderungen wie z.B. die einer Selbstständigkeit, Unsicherheit oder Instabilität in den Schatten stellt und an dessen Erhalt die Arbeiter*innen paradoxerweise aktiv beteiligt sind (vgl. ebd.). Viele YouTuber*innen haben sich bereits in einem Video oder in der Presse dazu geäußert, dass sie es problematisch finden, dass eine berufliche Tätigkeit auf YouTube nicht als eine, mit anderen Jobs, vergleichbare Tätigkeit gesehen wird oder dass eine Tätigkeit nur mit Spaß-haben assoziiert wird.

\section{Die Untersuchung}

\subsection{Forschungsfrage}

Welche systemischen, sozialen und emotionalen Herausforderungen gibt es für YouTuberinnen und YouTuber als Content Creator auf der Video-Plattform? Welche Rolle spielen hierbei das Individuum, die Gruppe und die Organisation und wie beeinflussen sich diese gegenseitig? Da YouTuber*innen nicht bei YouTube angestellt, sondern in selbstständiger Arbeit Videos produzieren und auf der Plattform hochladen, jedoch an Werbeeinnahmen beteiligt werden, ist der Einfluss des Systems auf die Herausforderungen des Einzelnen hier sehr interessant. Als soziales Netzwerk bietet YouTube nicht nur eine Plattform zum Ansehen von Videos, sondern auch einen Austausch der User und Creator untereinander. Daraus ergeben sich indirekte und direkte Kontakte, aus welchen auch sozialen Herausforderungen entstehen, auf die individuell emotional reagiert wird. Der Umgang und die Bewältigung von sozialen und emotionalen Herausforderungen im System YouTube liegen ebenso individuelle Strategien zugrunde.

\subsection{Forschungsdesign und methodisches Vorgehen}

Um die Untersuchungsfragen zu beantworten, wurden Methoden der qualitativen Sozialforschung gewählt (Lamnek und Krell 2016; Przyborski und Wohlrab-Sahr 2009). Zentrum der Arbeit bilden leitfadengestützte Experteninterviews mit YouTuber*innen aus verschiedenen Bereichen und ein Interview mit einer ehemaligen Produkt-Managerin von YouTube, um die Sichtweise der Organisation ebenfalls in die Arbeit einzubinden. Die transkribierten Interviews wurden anschließend mit der Methode der qualitativen Inhaltsanalyse ausgewertet und daraufhin anhand eines 
Kategoriensystems durchgearbeitet, welches unter Berücksichtigung der Fragestellung entwickelt wurde und das ,zentrale Instrument“ (Mayring 2015) der Inhaltsanalyse ist.

\subsection{Interviewpartner*innen}

Die befragten YouTuber*innen sind zwischen 21 und 32 Jahre alt und sind zwischen 11 Monaten und fünf Jahren auf YouTube aktiv (Stand April 2019). Vier der befragten Personen sind weiblichen und 2 männlichen Geschlechts und sind in unterschiedlichen Themenbereichen (Comedy, Politik, Lifestyle, Vlogging, Food, Gesellschaft) auf YouTube tätig. Die Gespräche zeichneten sich neben großer Offenheit auch durch eine sehr hohe Erzählbereitschaft, Erzähldichte und Sprachtempo aus. Der Auswahl der YouTuber*innen wurde eine Struktur zugrunde gelegt, welche sich auf die Abonnentenzahlen der befragten Personen bezieht. Für die Befragung wurden folgende drei Gruppen mit jeweils 2 Befragten gebildet: YouTuber*innen bis 10.000 Abonnenten, YouTuber*innen bis 100.000 Abonnenten und YouTuber*innen bis 500.000 Abonnenten (diese zwei sind hauptberuflich als Creator auf YouTube tätig). Anhand dieser Staffelung könnten zum Beispiel im Nachgang typische Entwicklungen im Karriereweg oder bestimmte Auffälligkeiten skizziert werden. Dieses Interview mit der ehemaligen Produktmanagerin zeichnete sich im Gegensatz zu den sechs anderen durch hohe Professionalität und auch durch mitunter lange Denkpausen aus, was vermutlich daran liegt, dass die Befragte aus ihrer Rolle, als Vertreterin der Organisation heraus berichtete.

\section{Ergebnisse}

Das in Kategorien sortierte und zusammengefasste Datenmaterial enthält neben einer Beschreibung der Aussagen der befragten Personen Originalzitate.

\subsection{Motive für die Aufnahme der Tätigkeit}

Die Niedrigschwelligkeit einen eigenen Kanal zu eröffnen, motiviert dazu es ,einfach einmal auszuprobieren“. „Und dann habe ich mir immer gedacht, ich habe da auch total Lust drauf, so einen kleinen Kanal zu machen“. Eine hauptberufliche YouTuberin sagt dazu: ,, und habe gedacht: ,Ach komm, das probierst du jetzt auch mal aus “". Mit anderen via YouTube in Kontakt zu treten und Gleichgesinnte $\mathrm{zu}$ finden, „(...) zu erzählen wie es mir so geht und was ich so denke über gewisse Sachen und ich hatte auch jetzt nicht so viele Freunde mit denen ich so viel gemacht habe oder so. Ich war eher Einzelgänger und fand die Vorstellung ganz toll, dass da draußen Leute sind, die vielleicht
Lust haben mir zuzuhören oder sich mit mir austauschen. “ und von Anderen Aufmerksamkeit zu erhalten wird in den Interviews häufig erwähnt. Die kreativen Ausdrucksmöglichkeiten, die sich durch das Medium Video bieten, sind ebenso ein genannter Grund. „Ich habe/Ich wollte irgendwie meine Kreativität/Ich wollte irgendwie mal mich publizieren. Das war so meine Intention. “, „Da hatte ich irgendwie Lust drauf, das irgendwie als kreatives Projekt zu sehen.“. Selbstausdruck spielt dazu auch eine Rolle. „Als ich damals begonnen habe war das für mich. Ich wollte mich, mein Gesicht, meine Person wollte ich halt raus in die Welt. Und dass Leute einfach wissen wer ich bin und da irgendwie Spaß haben “. Zwei YouTuber*innen, die zuvor in anderen Feldern tätig waren, sahen in YouTube eine Alternative zum herkömmlichen Karriereweg, des Berufszweiges. „Aber ich habe eben auch die Chance gesehen mich da zu verwirklichen und ich sage mal, mir eben selber eine Marke aufzubauen (...) Über YouTube war eben die Möglichkeit, wie gesagt GLEICH was zu machen. Natürlich nicht gleich darüber zu verdienen, aber SOFORT kreativ zu sein und mir peut á peut etwas aufzubauen. “. Wissen zu vermitteln oder eigene Gedanken mit anderen $\mathrm{zu}$ teilen, kommt ebenfalls zum Tragen. „ich mache super gerne politischen Content und ich finde es UNFASSBAR wichtig, das auch an junge Leute heranzutragen".

\subsection{Motivation für die Weiterführung der Tätigkeit/ Glücksmomente}

YouTube ist nicht nur eine Plattform, sondern eine Art Netzwerk, über das User untereinander und mit YouTuber*innen kommunizieren können. Daher bekommen Creator Feedback von den Zuschauenden, was als faszinierend und positiv beschrieben wurde. „Und ich habe positives Feedback bekommen von Menschen die ich nicht kannte. Das erste Mal in meinem Leben. So. Und das war so der Start, wo ich gesagt habe okay das will ich weiter machen, weil es mich in dem Moment erfüllt. “. Die befragten YouTuber*innen erhalten meist positives Feedback von ihren Zuschauer*innen für folgende Aspekte:

Einfluss im Leben der Zuschauer*innen (YouTuber*innen berichten übermäßig positiv, dass z. B. Zuschauer durch sie weniger Fleisch essen, wenn dies ihren eigenen Werten entspricht.), Identifikationsmöglichkeit (Wenn Zuschauer sich in den Videothemen der YouTuber*innen wiederfinden und sich hierfür z.B. bedanken, wird dies von den YouTuber*innen sehr emotional beschrieben.), Kommunikation/Interaktion (YouTuber*innen bekommen positives Feedback, wenn Sie viel mit ihren Zuschauern interagieren.), Leistung (Wenn YouTuber*innen regelmäßig Videos hochladen, wird das positiv wahrgenommen). Auffällig ist, dass kaum über konstruktives bzw. negatives Feedback 
gesprochen wurde, sondern viel mehr über abwertende Kommentare bzw. Hatespeech.

Durch die selbst produzierten Videos Menschen zu erreichen, zu berühren oder zu inspirieren, kurzum die eigene Wirksamkeit zu spüren, wurde von allen Befragten übermäßig positiv umschrieben. ,Wenn ich einfach merke, dass ich nicht nur auf Personen einen positiven Einfluss habe, sondern auch auf die Welt. Das wir damit was machen, was die Welt irgendwie ein Stück besser macht. Auch wenn das manchmal so ein bisschen abgedroschen klingt. “, „Das sind halt so (stockt) das sind so richtig krasse Dinge, die ich da bewege. Da kriege ich immer Gänsehaut und das ist so das allerschönste".

Der eigene Ermessensspielraum und die Freiheit spielen, vor allem bei den beiden hauptberuflichen YouTuberinnen, eine wichtige Rolle. „Zusätzlich ist halt, also außen rum ist es auch toll, dass ich mich weiter verwirklichen kann. (...) Und diese Chancen und diese Freiheit und der eigene Chef in diesem Bereich zu sein, sind so die Dinge die mich nach wie vor fesseln und (...) die ich nicht aufgeben will. “.

Der Kontakt mit den Personen, welche die Videos der Creator ansehen, wurde in allen Interviews sehr häufig als Glücksmoment genannt. Der Kontakt findet überwiegend indirekt, also über die Kommentarfunktion auf YouTube oder über andere Social-Media-Kanäle, dort ebenfalls über Kommentarfunktionen oder private Nachrichten statt. Auch die ehemalige YouTube Produktmanagerin beschreibt, dass der Kontakt zu den Abonnenten aus ihrer Erfahrung heraus, das Wichtigste für die YouTuber*innen ist und was durch verschiedene Funktionen auf YouTube möglich ist und ihrer Meinung nach auch verbessert werden könne. Die aus dem meist indirekten Kontakten entstehenden Beziehungen zu der eigenen Community oder Abonnenten wird als sehr nah und positiv beschrieben. Vier der sechs YouTuber*innen beschreiben eine Asymmetrie in der Kommunikation mit ihren Zuschauer*innen, die aufgrund des Mediums gegeben ist. „Das ist so das Verrückteste an der Sache glaube ich. Und das auch, dass man halt, du kennst mich, aber ich kenn dich nicht. (...) wo man natürlich auch selber daran schuld ist. Also, das ist ja klar, aber das ist verrückt.". Wenn die Creator im öffentlichen Raum auf ihre Zuschauer*innen treffen, beschreiben sie diese, für sie überraschenden Begegnungen, überwiegend positiv. Die zwei hauptberuflichen YouTuberinnen beschreiben beide jedoch auch Ausnahmen im direkten Kontakt, in denen sich Personen ihnen gegenüber merkwürdig oder aufdringlich verhielten und beschreiben, dass sie sich davon überrumpelt fühlten oder es ihnen sehr unangenehm war. Vier YouTuberinnen beschreiben auch Situationen in denen meist Mädchen und junge Frauen mit der Situation des Aufeinandertreffens überfordert schienen. „Also irgendwie verrückt, was man da für so ein übermenschliches Ding irgendwie angehängt kriegt, auf einmal, nur weil man irgendwie Videos von sich ins Internet stellt.". Eine nebenberufliche YouTuberin beschreibt auch den Erwartungsdruck der Zuschauer, den sie bei solchen Treffen wahrnimmt.

Drei von sechs befragten YouTuber*innen beschreiben konkret, wie sie sich deutlich mit ihren Zuschauer*innen identifizieren. „Die irgendwie so sind wie ich, letztendlich.“. „Es könnten irgendwie auch alle, es klingt so blöd, aber ich habe das Gefühl, man könnte auch irgendwie befreundet sein, oder die große Schwester je nachdem, wie alt die dann sind". Drei der sechs YouTuber*innen berichten auch von intimen Details, die Zuschauer*innen im Kontakt offenbaren. „Manche schreiben mir, dass sie sich ritzen und was sie machen sollen und dass sie Liebeskummer haben. Wirklich, das ist ganz/Man hat so viel Verantwortung auch. Manchmal beschwert mich das total, weil man so viel $z u$ tragen hat auch an den Problemen von anderen. ". Die YouTuber*innen schätzen die Intimität mit ihren Zuschauer*innen, benennen andererseits auch die Herausforderungen, die damit einhergehen.

\subsection{Herausforderungen der Tätigkeit}

Die Herausforderungen, die also solche, von den Interviewpartner*innen beschrieben werden, lassen sich in folgenden Bereichen zusammenfassen: Durchhaltevermögen, auf Work-Life-Balance und Psychohygiene achten, der Druck etwas produzieren zu müssen, sich die eigene Kreativität, $\mathrm{Spa} ß$ und Leidenschaft erhalten und den Einfluss der Systemlogik YouTube/Algorithmen. Diese Bereiche sind eng miteinander verknüpft, wie es in den folgenden Ankerbeispielen deutlich wird.

Einen erfolgreichen Kanal aufzubauen, kann im Durchschnitt ca. 3 bis 5 Jahre dauern und braucht Durchhaltevermögen und Frustrationstoleranz. „Ja, wie das bei YouTube immer so ist. Also relativ schleppend am Anfang, also da braucht man immer einen langen Atem. ". In dieser Zeit, muss der Lebensunterhalt der Person, über andere Einkommensquellen gesichert werden. „Ach so ja, eben Fuß zu fassen eigentlich im Prinzip, weil wenn man mit der Ausgangslage startet, man möchte das zum Beruf machen, glaube ich, dass es viele sehr frustriert und dass sie nicht lange durchhalten, (...) da dauert es drei bis vier Jahre, bis sie mal $1000 €$ überhaupt verdienen. ". Die drei befragten YouTuber*innen mit den meisten Abonnenten berichten über Herausforderungen in der Work-Life-Balance. „Ja du kannst halt, du kannst einfach theoretisch gesehen 24/7 arbeiten" Die zwei hauptberuflichen YouTuberinnen berichten von einem Burnout oder Erschöpfungszuständen, die sie mittlerweile überwunden haben. „Und so war es schlussendlich dann auch, dass ich eben mindestens 5 Tage die Woche gedreht habe, ich habe aber vergessen, dass man ja die Drehs auch vorbereiten muss. Sprich ich 
habe, dann auch die restlichen zwei Tage, Samstag, Sonntag, die Drehs vorbereitet und entsprechend war ich nach einem Jahr, eineinhalb Jahren, also nach einem Jahr habe ich es dann gemerkt. (...) Und krank bin ich auch geworden. “. Fünf der sechs befragten YouTuber*innen sprachen über einen Druck, kreativ sein zu müssen, auch wenn die keine intrinsische Motivation verspürten, was als Pressure to create bezeichnet wird. ,, und was noch eine Sache ist, ist halt wo jeder YouTuber auch struggelt, ist eben dieses kreativ sein. (...) manchmal sprudelt es nicht aus dir heraus und du musst dir trotzdem manchmal irgendwas aus den Fingern saugen. Und das ist SCHEISSE manchmal. (...) also irgendwas muss ich bringen, WEIL (...) der Algorithmus, was eigentlich im Endeffekt ja nur die Zuschauer sind, sind es gewohnt einmal die Woche ein Video zu bekommen und du wirst eben auch sehr schnell VERGESSEN, wenn du nicht deinen Rhythmus einhältst.“.

Die beiden YouTuber*innen mit weniger Abonnenten als die anderen vier, erwähnen „den YouTube-Algorithmus“ oder Systemlogiken häufiger als Herausforderung. ,Herausforderungen sind meiner Meinung nach, wenn man eben diesen YouTube Algorithmus mit ihm arbeiten willst. Also absolut das alles ist wofür du dann arbeitest. Aber dafür, dass $d u$ da halt viel von deiner eigenen Kreativität und Persönlichkeit verlierst. Du musst da natürlich abwiegen wie sehr möchtest $d u$ wirklich ein Teil von YouTube sein oder wie sehr möchtest du ein Teil von dir sein, wo du YouTube als Medium benutzt. “. Auch der Umgang mit den SystemRegeln, an welche man sich halten muss, werden kritisch betrachtet: ,aber dafür können Sie ja so ein - wie auch immer dieses Teil heißt-dass das scannt irgendwie einrichten, aber doch nicht (...) also das finde ich irgendwie krass, dass man da auf- man muss aufpassen, was man sagt. Eben, ich sage schon oft Scheiße und so. Ich muss das jedes Mal rausschneiden, und das ist schon irgendwie eine Art von Einschränkung, die man hat.".

Die ehemalige YouTube Produkt-Managerin sieht die Herausforderungen für YouTuber*innen wie folgt: „It's just getting the equipment, having the time in the day, figuring out what to, what to record, what they have to edit, some maybe don't know how and then there are a lot of complexities right. You add music, you select music that isn't copyrighted, if you do select copyrighted music only for a short period of time, these are all the things that the tools help you with right. " Außerdem ist die Konkurrenz auf YouTube und dem damit verknüpften Markt groß. „(...) and so 10 years ago was relatively easy to be discovered on YouTube and now because there is so much content it's a bit harder for new creators to be discovered. "

\subsection{Widersprüche}

In einem Widerspruch schließen sich, nach der dialektischen Methode, These und Antithese nicht aus (Störig 1992). Diese Haltung bestimmt die Sichtweise auf Widersprüche im System YouTube in der vorliegenden Arbeit und betrachtet einen Widerspruch als Koexistenz von These und Synthese. „Ich finde YouTube ist ein gleichzeitig wunderschöner, kann aber auch ein grausamer Ort sein. Oder generell das Internet oder Social Media. Da muss man aufpassen. Und gleichzeitig auch nicht, finde ich. Also das ist ganz weird.". Diese Aussage eines befragten YouTubers bildet ab, dass YouTube als System sehr unterschiedliche Facetten hat und diese Facetten auch als widersprüchlich wahrgenommen werden. Die Interviewpartner*innen schilderten in den Interviews verschiedene Widersprüche, die ihnen in ihrer Tätigkeit auf YouTube begegnen. Für diesen Artikel werden drei Grund-Widersprüche von sieben angeführt.

\subsubsection{Reallife - Virtuallife}

Die Widersprüchlichkeit zwischen physischem Leben (Reallife) und dem Leben in der virtuellen Welt Internet verdeutlicht sich und kann zum Beispiel die Wahrnehmung der eigenen Person umfassen. „Ich bin eigentlich so ein total schüchterner Mensch und introvertiert (...). Und auf YouTube denkt man nur, weil ich da viel rede und immer mich zeige bin ich auch im normalen Leben so, aber das ist manchmal ein falsches Bild. Und man macht sich ja auch immer von Leuten diese Bild, wie sie aussehen. Das ist ja wie, wenn man ein Buch liest. Man macht seine Welt darum. ". Die Existenz der eigenen Person im Reallife und im Virtuallife kann auch Beziehungen im Reallife beeinflussen. „Weil man macht sich ein Bild von der Person (...) ungefragt. Also ein ungewolltes und klar, vielleicht ist es dann anders, und dann clasht das so aufeinander, und du hast vielleicht Erwartung an die Person, die gar nicht der Realität entsprechen. (...) Ja, was irgendwie verrückt ist, weil - das bin ja schon alles ich". Dieser Widerspruch hängt eng mit dem Folgenden zusammen, da gerade zwischen öffentlich und privat das Internet eine große Rolle spielt.

\subsection{2 Öffentlich - Privat}

Dieser Widerspruch wird in der Schilderung einer nebenberuflichen YouTuberin deutlich. Wenn in Videos zum Beispiel private Informationen zu sehen sind oder aus dem Video die Adresse der Person abgeleitet werden kann, hat das eine Auswirkung auf das Wohlbefinden der Person. „Das war richtig creepy. Und seitdem muss ich aufpassen, dass ich nie mein Fenster filme, weil du auf allen Seiten siehst: Da sind die Cafés, da siehst du ja wo ich wohne, hier ist 
die Bären Apotheke. (...) Also was machst du, wenn du dich zu Hause nicht mehr sicher fühlst? Das ist natürlich ganz übertrieben. Aber gleichzeitig ist es halt so, die weiß (...) die Person weiß alles andere auch über mich. Also Nein, aber so viele Sachen. Irgendwie ist es crazy. “. Die befragten YouTuber*innen ziehen unterschiedliche Grenzen, was ihr Privatleben betrifft. Gleichzeitig offenbaren sie zum Beispiel ihre Gefühle oder zeigen ihren Tagesablauf oder privaten Räume. Außerdem müssen YouTuber*innen, die kein Büro oder Management haben, aufgrund von geltendem Recht im Impressum ihre Adresse veröffentlichen.

\subsubsection{Freiheit - Reglement}

Die befragten YouTuber*innen schätzen YouTube als Plattform, auf der sie sich kreativ ausprobieren können. Die vier Wertesäulen von YouTube ${ }^{15}$ umfassen drei Freiheiten: Meinung, Information, Zugehörigkeit. Gleichzeitig gibt es einschränkende Richtlinien für Personen und Inhalte auf YouTube, wie vulgäre Sprache, Nacktheit oder sexuelle Inhalte, schädliche Inhalte, etc. ${ }^{16}$. Diese Richtlinien sollen „das Miteinander auf der Plattform regulieren“. Die häufige Benutzung bestimmter Wörter ist laut den Richtlinien von YouTube verboten. „Du darfst bestimmte Wörter nicht sagen, du fällst dann halt erstmal raus aus überhaupt dem Suchmechanismus. Also bei YouTube wirst du dann nicht angezeigt. Und du kannst aber kein Geld verdienen. (...) Und das erkennt YouTube halt recht gut. Ja das ist blöd. Also dann richtest du dich halt danach und das ist eigentlich nicht das was ich/was man tun sollte. Dass du da halt sitzt und sagst oh warte nein das sollte ich so machen, weil sonst mag mich YouTube nicht. So. Und das ist halt nicht richtig. ". Auch bestimme Video-Themen (z.B. Menstruation, sexuelle Aufklärung, LGBTIQ $+{ }^{17}$ ), werden nach den Aussagen der befragten YouTuber*innen als nicht-familyfriendly gekennzeichnet und somit von der Monetarisierung ausgeschlossen.

\section{Diskussion und Interpretation der Ergebnisse}

Im folgenden Abschnitt folgen drei Annahmen für mögliche Ursachen der beschriebenen Herausforderungen und Hindernisse. Es wird aufgezeigt, weshalb die Tätigkeit gleichzeitig als attraktiv wahrgenommen wird.

\footnotetext{
$15<$ https://www.youtube.com/intl/de/yt/about/> [16.05.2020].

$16<$ https://www.youtube.com/intl/de/yt/about/policies/\#communityguidelines $>$ [16.05.2020].

17 LGBTIQ+: Lesbian, Gay, Bisexual, Trans-, Intersexual, Queer als Bezeichnung für eine nicht-binäre geschlechtliche und/oder sexuelle Identität.
}

\subsection{Die Selbstreferenz in der Tätigkeit}

Durch die Beschreibung des Arbeitsprozesses der Interviewpartner*innen wurde deutlich, dass der endgültigen Videolänge ein langer Arbeitsprozess vorangeht, in dem die YouTuber*innen kurzgefasst ein Video konzipieren, drehen und schneiden. In 5 von 6 Fällen können die befragten YouTuber*innen als ein Ein-Personen-Team gesehen werden. Das heißt, dass die befragten Personen während dieses Prozesses, wenn sie diese Arbeit allein tun, mit sich selbst konfrontiert sind. Ihre Motivation ist intrinsisch, sie schöpfen aus sich selbst heraus Ideen für die Videos. Während des Drehens sprechen sie in eine Kamera und sehen oder spiegeln sich im Bildschirm der Kamera oder in der Kameralinse. Während des Videoschnitts sehen und hören sich die YouTuber*innen über Stunden hinweg selbst, da der Videoschnitt mitunter der langwierigste Arbeitsprozess ist. Sie sind also mit ihrer eigenen Person auf konzeptioneller und audiovisueller Ebene Teil des eigenen Arbeitsprozesses und selbst der Hauptfokus (im wahrsten Sinne des Wortes) ihrer Tätigkeit. Fosatti beschreibt YouTube als Mirror-Maze (engl. Spiegel-Labyrinth). „When looking at your computer screen with the webcam on, you are looking at your own reflection. YouTube reflects you and you reflect (on) YouTube. On the other side of the mirror, all YouTubers are watching." (Fossati 2009, S. 460). Er beschreibt damit eine Metapher (YouTube als Spiegellabyrinth oder Spiegelkabinett), das auf gleiche Weise fasziniert und überfordert.

Die Entfaltung des Individuum ist seit der Aufklärung in den Mittelpunkt des west-europäischen gesellschaftlichen Bewusstseins gerückt (vgl. Kant 2016). Die Technologien des Selbst (vgl. Foucault 2009) sind ein wichtiger Teil in der Konstruktion des Subjektbegriffs der Medientheorie und -philosophie der Gegenwart. Die Konstruktion des Subjekts hat durch Massenmedien und Soziale Medien eine unfassbare Anzahl an Möglichkeiten erhalten. Das Ich als Marke (vgl. Adlmaier-Herbst 2011), also als marktfähiges Produkt, ist in der westlich kulturell geprägten Welt ein zentrales Thema geworden. „Sich selbst zu verkaufen“ spielt nicht nur in Bewerbungsverfahren eine Rolle, sondern stellt eine tagtägliche Repetition dar (Bublitz 2010). Das heißt, dass nicht das Individuum, um zurück zu Fosatti's Spiegel-Metapher zu kommen, sich ständig spiegeln will, sondern dass es schwer fällt sich in den unendlichen medialen Möglichkeiten und unter dem sozioökonomischen Druck (vgl. ebd.) nicht zu spiegeln. In der Spiegelung vergewissert sich das Individuum laut Bublitz sich seiner Existenz.

Die Motivation, die sehr deutlich in den Interviews zum Tragen kam, war die Kommunikation mit anderen und ein erhöhtes Sendungsbewusstsein. Ein Faktor, den alle befragten YouTuber*innen als übermäßig positiv beschrieben haben, ist die Wirksamkeit (andere inspirieren, berühren, er- 
reichen), die sie durch ihre Videos und über die Kommentare in den Zuschauer*innen spüren. Dieses Phänomen wird als Kontrollüberzeugung, also der Idee, dass man selbst etwas beeinflussen kann, als Teil des Konzepts der Selbstwirksamkeitserwartung nach Bandura (vgl. Bandura 2010; Batinic und Appel 2008) beschrieben. Die Motivation, sich selbst in einem Video „zu senden“, liegt hier in der möglichen Kontaktaufnahme mit anderen und einem erwarteten Feedback. In einer Studie der Harvard Universität aus dem Jahre 2012 legten die Forscher*innen dar, dass die Selbstoffenbarung einen belohnungsartigen Effekt auf Menschen hat, was auf die Sozialität der menschlichen Spezies zurückgeführt wird (vgl. Tamir und Mitchell 2012). Andere Studien, deren Ergebnisse in der genannten Studie dargelegt wurden, sagen aus, dass mögliche Gründe für Selbstoffenbarung von Menschen die Erwartung einer Gegenerwiderung, Vorteile für das persönliche Wohlbefinden und eine gesteigerte Sympathie zwischen Beziehungspartnern sein können (vgl. Collins und Miller 1994). Die technischen und medialen Möglichkeiten, die durch die Entwicklungen von tragbaren Devices und Social Media /Sozialer Netzwerke entstanden sind und entstehen, erhöhen die Möglichkeiten der täglichen Kontaktaufnahme, Kommunikation und Selbstoffenbarung enorm.

Eine in den Interviews genannte Herausforderung ist „sich selbst nicht zu verlieren“ oder ,,sich selbst treu zu bleiben“. Hier wird, ähnlich wie im Umgang mit der Kritik, ein ähnliches Auf-Distanz-Gehen beobachtet. Die Formen dieser Coping Reaktionen (Längle und Bürgi 2014) drücken sich bei Menschen unterschiedlich aus. Sie führen dazu, dass die Person auf Distanz zu anderen, dem Gegenüber oder einem bestimmten Sachverhalt geht. Sie ist auf sich zurückgeworfen. In einer virtuellen Welt oder im System YouTube auf sich selbst zurückgeworfen zu sein mag andere Verarbeitungsstrategien verfolgen, als in einem physischen Raum.

Die erste Annahme, die hieraus hervorgeht, ist, dass die YouTuber*innen aufgrund der im System verankerten Selbstreferenz in ihrer gegebenen Selbstdistanzierung und Selbsttranszendenz (vgl. Frankl 2018) eingeschränkt sind und dies eine der Ursachen für die benannten Herausforderungen darstellt. ,Je mehr er [Anm. der Mensch] sich selbst übersieht, je mehr er sich selbst vergißt, in dem er sich hingibt einer Sache oder anderen Menschen, desto mehr ist er Mensch, desto mehr verwirklicht er sich selbst.“ (Frankl 1983, S. 110).

\subsection{Die Vereinzelung in der Tätigkeit}

Die befragten Personen äußerten sich alle positiv über ihre Freiheiten und die Unabhängigkeit, die sie im Arbeitsformat der „Selbstständigkeit“" als Content Creator haben. Sie benannten jedoch Schwierigkeiten im Zeitmanagement und der Work-Life-Balance, sowohl die Abhängigkeit von den Funktionen und Wirkungsweisen der Algorithmen als Herausforderungen. Dieses Paradox wird von Muckenhuber als „Autonomieparadox“ beschrieben (Muckenhuber 2014). In der durchgeführten Untersuchung mit Selbstständigen wurde ein Spannungsfeld zwischen der freien Verfügbarkeit der Zeit und den realen Anforderungen der Tätigkeit sichtbar. Eine befragte Person gab an, dass ihr allein schon der Gedanke theoretisch zwei Tage frei machen zu können reiche, obwohl dies in ihrem Arbeitsalltag nie möglich sei (vgl. ebd.). Selbstständig tätige Personen sind für ihr Zeitmanagement, Aufgabenmanagement und für ihren Erfolg selbst verantwortlich.

Zwei befragte YouTuberinnen berichten von Erschöpfungszuständen und Burnout-Symptomatiken. Ein Befragter arbeitet zusätzlich zu seinem 40-Stunden-Job noch ca. $20 \mathrm{~h}$ an seinen YouTube Videos. Der Faktor Zeit wurde von allen drei als maßgeblich für die Überarbeitung genannt. In selbstständiger Tätigkeit greifen Überstundenregelungen, Krankheits- oder Schwangerschaftsausfälle und andere Arbeitszeitmodelle nicht, um die Erwerbstätigen zu entlasten. Zudem besteht keine, im System verankerte, dringende Notwendigeit zur Zusammenarbeit. Dies begründet sich auch daraus, dass jede Person ihren eigenen Kanal betreibt und ihre eigene Zuschauerschaft hat.

Zwischen den befragten YouTuber*innen und ihrer Zuschauerschaft besteht eine asymmetrische Kommunikation. Die YouTuber*innen nehmen sich als ,normale“ Menschen wahr und äußerten sich verwundert, warum Zuschauer*innen z.B. hysterisch reagierten, wenn sie diese im öffentlichen Raum trafen. Die Schwierigkeit für die YouTuber*innen liegt darin, diese Lücke zwischen der eigenen Wahrnehmung und der Zuschauerschaft zu überbrücken. Im Gegensatz zu z. B. Musiker*innen, findet der Kontakt zu Zuschauer*innen nur virtuell (über Kommentare, Likes, Dislikes etc.) statt. Die YouTuber*innen spüren die Menge ihrer Fans nicht, wie z.B. Musiker*innen die auf einem Festival Menschenmassen mit der Musik unterhalten. Die Zahl der Abonnenten ist abstrakt, nicht greifbar. Dieses Phänomen wird als parasozialer Kontakt und die, aus diesem Kontakt entstehende Beziehung, als parasoziale Beziehung beschrieben (Wirtz 2014). Die sogenannte Persona (eine Art Rolle einer Persönlichkeit) unterscheidet sich durch ihre Alltäglichkeit und Nahbarkeit von klassischen Stars. Ein Beziehungsaufbau scheint möglich, ist jedoch eine Illusion, da der Abstand per se nicht überwunden werden kann (Hartmann 2016).

Neben der parasozialen Beziehung wurde ein zweites Phänomen beobachtet. In den Interviews beschrieben vier der Befragten, mit den meisten Abonnenten ihre Zuschauer*innen übermäßig positiv und sprachen gleichzeitig davon, wie ähnlich sie ihren Zuschauer*innen wären. Dieses Phänomen ist als „Zwillingsübertragung“ beschrieben 
(Stumm und Voracek 2009), welches von Heinz Kohut im Konzept der Selbstobjektübertragung (Kohut und Elson 1993) entwickelt wurde. Mit der Zwillingsübertragung auf das idealisierte Gegenüber, hat die Person die Möglichkeit sich gleichwertig und akzeptiert zu fühlen. Eine Aufwertung des Gegenübers hat eine Aufwertung des Selbst zur Folge (Stumm und Voracek 2009). Wie stehen die parasoziale Beziehung und die Zwillingsübertragung nun miteinander in Verbindung? Die Zuschauer*innen fühlen sich durch die parasoziale Beziehung in einer gleichwertigen Beziehung zu den YouTuber*innen. Die Zwillingsübertragung der YouTuber*innen auf der anderen Seite könnte den Versuch markieren, die für sie vorhandene Asymmetrie der Beziehung auszugleichen.

Ein interessantes Phänomen, welches aus den Ergebnissen der Untersuchung hervorging ist, dass die zwei YouTuber*innen mit unter 10.000 Abonnenten am meisten Kritik am System und der Organisation YouTube äußerten. Sie verknüpften ihre Herausforderungen direkt mit den von YouTube gestalteten Mechanismen oder Algorithmen. Wohingegen die beiden hauptberuflichen YouTuberinnen dahingehend wenig Kritik an YouTube äußerten. An dieser Stelle wäre es unbedacht, das System YouTube als nicht Teil der Vereinzelungstendenz in einem größeren gesellschaftlichen Zusammenhang zu betrachten. Ein befragter YouTuber findet, dass es vermessen sei, YouTube an dieser Stelle zu kritisieren, denn YouTube wäre keine Wohltätigkeitsorganisation, sondern ein gewinnorientiertes Unternehmen. Wenn man sich nicht an die Spielregel halten wolle, müsste man sich eine andere Plattform suchen. YouTube ist unangefochtener Marktführer als Online-Video-Portal. Es gibt kaum andere Video-Plattformen, die an die Relevanz von YouTube heranreichen könnten und somit eine Alternative bieten. Hier zeigt sich deutlich ein neoliberales Motiv (hier ist der kritische Gebrauch dieses Begriffes beabsichtigt z.B. nach Chomsky (2000)).

„Das neoliberale Subjekt seiner selbst ist nicht fähig zu Beziehung zu anderen, die frei vom Zweck wären. Zwischen Unternehmern entsteht auch keine zweckfreie Freundschaft. Frei-sein bedeutet aber ursprünglich bei Freunden sein. Freiheit und Freund haben im Indogermanischen dieselben Wurzeln. [...] Die totale Vereinzelung, zu der das neoliberale Regime führt, macht uns nicht wirklich frei.“ (Han 2014, S. 11). Nach Han steckt die Freiheit in der Krise. Das Subjekt wähnt sich frei, ist aber unfrei, da es sich selbst innere Zwänge, wie Leistungs- und Optimierungszwang auferlegt. Depression und Burnout sind für Han „der Ausdruck einer tiefen Krise der Freiheit“ (vgl. ebd., S. 10). Burnout hängt mit der Arbeitsform und dem System zusammen.
In einem Video ${ }^{18}$ zum Thema Creator-Burnout auf dem YouTube eigenen Kanal YouTube Creators wird Elle Mills gegen Ende des Videos vom Interviewleiter gefragt: „(...) but is there anything that we should do to help with this issue as a platform?" Auf diese geschlossene Frage, verneint Elle Mills nach kurzem Zögern die Frage und meint, dass es von YouTubes Seite ausreiche über das Thema zu sprechen. Gegen Ende des Videos erklärt Elle Mills auf die Frage, wie es ihr besser gehen könnte: „A lot of it is a ,me problem, and I think that's working on myself and taking the time to work on myself. And getting extra help, like therapy obviously. “. Elle Mills gibt vor, keine Verantwortung des Systems oder der Organisation YouTube zu sehen. Burnout ist für sie ein individualisiertes Problem. YouTube schlägt YouTuber*innen vor, wenn sie erste Anzeichen eines Burnouts bemerken würden, eine Auszeit von YouTube und professionelle Hilfe in Anspruch zu nehmen ${ }^{19}$. Vorschläge wie der damit verbundene Arbeitsausfall (Existenzangst) und die Kosten für z. B. therapeutische Hilfe o. ä. ausgeglichen werden können, gibt es bislang nicht.

Die zweite Annahme, die hier hervorgeht und mit der ersten Annahme verwoben ist, ist, dass es für YouTuber*innen in ihrer Tätigkeit, aufgrund der systemimmanenten Strukturen, Phänomene und Prozesse schwierig ist, die Vereinzelung zu überwinden.

\subsection{Das Alleinsein in der Tätigkeit}

Hier muss zunächst der Begriff Alleinsein definiert und vom negativ konnotierten Begriff der Einsamkeit abgegrenzt werden. Alleinsein im Kontext von YouTube bedeutet zunächst, dass es möglich ist, allein eine Bühne, einen Kanal $\mathrm{zu}$ bespielen und die eigenen Ideen, ohne Limitation von außen, umzusetzen. Eine befragte YouTuberin gibt an, dass sie eigentlich eine eher introvertierte Person sei und dass es deshalb für sie einfach sei, auf YouTube von sich eine andere Seite zu zeigen. Eine hauptberufliche YouTuberin sieht sogar ökonomische Vorteile, dass sie in einer bestimmten Region in Süddeutschland ein Alleinstellungsmerkmal hat. Sie beschreibt gleichzeitig ein Einsamkeitsgefühl, das auch schon von anderen YouTuber*innen in öffentlichen Videos beschrieben wurde. Dieses Einsamkeitsgefühl geht vor allem aus der Vereinzelung in der Tätigkeit hervor und wird von der Selbstreferenz und der Parasozialen Beziehung verstärkt. Zur Frage, ob übermäßige Medien- und Internetnutzung Einsamkeitsgefühle verstärkt, liegen in der

\footnotetext{
18 YouTube Creators: „Elle Mills zum YouTuber-Burnout I Creator Coffees" vom 05.02.2019<https://www.youtube.com/watch? $\mathrm{v}=$ seCudUlxehs $>$ [16.05.2020].

19 YouTube Creators Academy: „Auf das eigene Wohlbefinden achten und Burn-out vermeiden“ vom 05.06.2018<https://creatoracademy. youtube.com/page/lesson/self-care?cid=well-being\&hl=de $>\quad[16.05$. 2020].
} 
aktuellen Forschung widersprüchliche Ergebnisse vor (vgl. Batinic und Appel 2008).

Die dritte Annahme, die hier hervorgeht, ist, dass YouTuber*innen durch das Alleinsein in der Tätigkeit Einsamkeitsgefühle erleben können, wenn sie dies hauptberuflich und überwiegend, als ein-Personen-Produktionssystem ausführen.

Zusammengefasst heißt das, dass durch die Selbstreferenz der Tätigkeit, die Vereinzelung in der Tätigkeit und das Alleinsein in der Tätigkeit, die Herausforderungen wie Überforderung im Zeitmanagement, Stress, Umgang mit negativem Feedback, der Abhängigkeit von der Organisation, der Kontakt zu den Zuschauer*innen, als individualisierte Probleme wahrgenommen werden. Diese Wahrnehmung kann das bereits geschilderte Einsamkeitsgefühl noch verstärken.

\section{Fazit und Ausblick}

In der Untersuchung konnte dargelegt werden, dass YouTuberinnen und YouTuber in ihrer Tätigkeit als Content Creator auf YouTube mit einem hohen Maß an systemischen, emotionalen und sozialen Belastungen konfrontiert sind. Diese Belastungen führen unter bestimmten Umständen zu einem Erschöpfungszustand oder zum Burnout. Belastungen sind eine unausgeglichene Work-Life-Balance; die Schwierigkeiten in der Selbstorganisation und im Zeitmanagement im selbstständigen Arbeiten; das Alleinsein und der fehlende Kontakt zu Kolleg*innen; die Ansprüche der Zuschauer*innen an die eigene Arbeit; die Abhängigkeit von der Systemlogik und den Wirkungsweisen der Algorithmen; die nachhaltige Bindung der Zuschauerschaft mithilfe von authentischem Auftreten; der Druck, etwas produzieren zu müssen (pressure to create); die Öffentlichkeit und der Verlust der Privatsphäre, die mit einer Tätigkeit auf YouTube einhergeht; (mitunter übermäßiges) negatives Feedback der Zuschauerschaft. Die Ursachen dieser Herausforderungen liegen in der Selbstreferenz der Tätigkeit, die Vereinzelung in der Tätigkeit und das Alleinsein in der Tätigkeit, die diese Herausforderungen, als individualisierte Probleme darstellen.

An dieser Stelle soll auf die Limitation der Arbeit hingewiesen werden. Die geringe Anzahl an Interviews lässt keine allgemein gültigen Aussagen zu. Zukünftige Studien könnten mit quantitativer Forschung die hier getätigten Annahmen überprüfen. Einer Differenzierung hinsichtlich der Größe der Plattform und der Anzahl der Abonnenten, die sich in den Interviews andeutet, könnte ebenfalls empirisch mehr Aufmerksamkeit gewidmet werden.

Es handelt sich hier mitnichten um ausschließlich individuelle Belastungen, sondern um medientechnologische Umbrüche, sozioökonomischen Veränderungen und Sub- jektivierung der Arbeitswelt, die dazu geführt haben, dass die Gruppe in dieser Art von Tätigkeit, als Faktor zwischen Individuum und Organisation, weniger vorhanden ist. Das Individuum steht der Organisation allein gegenüber und Vergemeinschaftung von Problemen und Fragestellungen finden aufgrund der systemimmanenten Strukturen weniger statt.

Für die Zukunft ist es von großer Wichtigkeit, sowohl die Organisation des Einzelunternehmertums YouTube, als auch die Aus- und Weiterbildungsmöglichkeiten dieser Tätigkeit weiterzuentwickeln. Durch Ausbildungsorganisationen und die Installation eines Berufsverbandes würde die Reputation und die rechtliche und politische Handhabe der YouTuber*innen gestärkt werden. Dort könnten die Arbeitsbelastungen thematisiert und arbeitsweltliche Beratungsangebote unabhängig verankert und angeboten werden. Auch hinsichtlich des Arbeitsrechtes müsste geklärt werden, inwiefern YouTube durch das Partnerprogramm und die daraus entstehende monetäre Vergütung und Weisungsgebundenheit (Einhalten von Richtlinien), Verantwortung für YouTuber*innen übernehmen muss. Auch wäre es denkbar, dass YouTuber*innen als kreativ, künstlerisch tätige Personen in die Künstlersozialkasse aufgenommen werden könnten und YouTube als Plattform, für die die Videos produziert werden, sich an den Beiträgen beteiligt.

Funding Open Access funding enabled and organized by Projekt DEAL.

Open Access Dieser Artikel wird unter der Creative Commons Namensnennung 4.0 International Lizenz veröffentlicht, welche die Nutzung, Vervielfältigung, Bearbeitung, Verbreitung und Wiedergabe in jeglichem Medium und Format erlaubt, sofern Sie den/die ursprünglichen Autor(en) und die Quelle ordnungsgemäß nennen, einen Link zur Creative Commons Lizenz beifügen und angeben, ob Änderungen vorgenommen wurden.

Die in diesem Artikel enthaltenen Bilder und sonstiges Drittmaterial unterliegen ebenfalls der genannten Creative Commons Lizenz, sofern sich aus der Abbildungslegende nichts anderes ergibt. Sofern das betreffende Material nicht unter der genannten Creative Commons Lizenz steht und die betreffende Handlung nicht nach gesetzlichen Vorschriften erlaubt ist, ist für die oben aufgeführten Weiterverwendungen des Materials die Einwilligung des jeweiligen Rechteinhabers einzuholen.

Weitere Details zur Lizenz entnehmen Sie bitte der Lizenzinformation auf http://creativecommons.org/licenses/by/4.0/deed.de.

\section{Literatur}

Adlmaier-Herbst, D. G. (Hrsg.). (2011). Der Mensch als Marke: Konzepte, Beispiele, Experteninterviews. Göttingen: Business Village.

Bandura, A. (2010). Self-efficacy: the exercise of control. New York: Freeman.

Batinic, B., \& Appel, M. (2008). Medienpsychologie. Berlin, Heidelberg: Springer.

Bublitz, H. (2010). Im Beichtstuhl der Medien: die Produktion des Selbst im öffentlichen Bekenntnis. Bielefeld: transcript. 
Burgess, J., \& Green, J. (2009). The entrepreneurial vlogger: Participatory culture beyond the professional-amateur divide. In P. Snickars \& P. Vonderau (Hrsg.), The YouTube Reader. Stockholm: National Library of Sweden.

Burgess, J., \& Green, J. (2018). Youtube - Online video and participatory culture. Bd. 2. Cambridge: Polity Press.

Chomsky, N. (2000). Profit over people: Neoliberalismus und globale Weltordnung. Hamburg: Europa-Verlag.

Collins, N.L., \& Miller, L.C. (1994). Self-disclosure and liking: A meta-analytic review. Psychological Bulletin, 116(3), 457-475. https://doi.org/10.1037/0033-2909.116.3.457.

Duffy, B., \& Wissinger, E. (2017). Mythologies of creative work in the social media Age: fun, free, and "Just being me.". International Journal of Communication, 11, 4652-4671.

Fossati, G. (2009). Youtube as a mirror maze. In P. Snickars \& P. Vonderau (Hrsg.), The youtube reader. Stockholm: National Library of Sweden.

Foucault, M. (2009). Hermeneutik des Subjekts: Vorlesungen am Collège de France (1981/82). Frankfurt am Main: Suhrkamp.

Frankl, V.E. (1983). Das Leiden am sinnlosen Leben: Psychotherapie für heute (7. Aufl.). Freiburg: Herder.

Frankl, V.E. (2018). Der leidende Mensch: anthropologische Grundlagen der Psychotherapie. Bern: Hogrefe.

Han, B.-C. (2014). Psychopolitik: Neoliberalismus und die neuen Machttechniken. Frankfurt am Main: Fischer.

Hartmann, T. (2016). Mass communication and para-social interaction: observations on intimacy at a distance. In M. Potthoff (Hrsg.), Schlüsselwerke der Medienwirkungsforschung (S. 75-84). Wiesbaden: Springer.

Jahnke, M. (Hrsg.). (2018). Influencer Marketing: Für Unternehmen und Influencer: Strategien, Plattformen, Instrumente, rechtlicher Rahmen. Wiesbaden: Springer.

Kant, I. (2016). Was ist Aufklärung? North Charleston: CreateSpace Independent Publishing Platform.

Kohut, H., \& Elson, M. (1993). Auf der Suche nach dem Selbst: Kohuts Seminare zur Selbstpsychologie und Psychotherapie mit jungen Erwachsenen. München: Pfeiffer.

Lamnek, S., \& Krell, C. (2016). Qualitative Sozialforschung. Weinheim: Beltz. mit Online-Material

Lange, P. (2007). Commenting on comments: investigating responses to antagonism on youtube

Längle, A., \& Bürgi, D. (2014). Existentielles Coaching: theoretische Orientierung, Grundlagen und Praxis für Coaching, Organisationsberatung und Supervision. Wien: Facultas.

Lohmaier, J., \& Schäfer, F. (2016). Youtuber als Beruf: Hallo, ihr Lieben. Süddeutsche Zeitung. https://www.sueddeutsche.de/karriere/ job-youtuber-als-beruf-hallo-ihr-lieben-1.3214159 (Erstellt: 22. Okt. 2016). Zugegriffen: 16. Mai 2020.
Marek, R. (2013). Understanding YouTube: Über die Faszination eines Mediums. Bielefeld: transcript.

Mayring, P. (2015). Qualitative Inhaltsanalyse - Grundlagen und Techniken (12. Aufl.). Weinheim: Beltz.

Meyer, M. (2019). Die Disruption der Sehgewohnheiten Wie man mit Online-Videos auf YouTube und anderen Plattformen die Zuschauer erreicht. In H. Haarkötter \& J. Wergen (Hrsg.), Das YouTubiversum. Wiesbaden: Springer.

Muckenhuber, J. (2014). Arbeit ohne Ende?: Zur Arbeitsrealität der „neuen“ Selbstständigen. Konstanz: UVK.

Opresnik, M. O., \& Yilmaz, O. (2016). Die Geheimnisse erfolgreichen YouTube-Marketings: Von YouTubern lernen und Social Media Chancen nutzen. Berlin, Heidelberg: Springer.

Przyborski, A., \& Wohlrab-Sahr, M. (2009). Qualitative Sozialforschung: ein Arbeitsbuch. München: Oldenbourg.

Rieder, B., Matamoros-Fernández, A., \& Coromina, Ò. (2018). From ranking algorithms to "ranking cultures": Investigating the modulation of visibility in YouTube search results. Convergence, 24(1), $50-68$.

Störig, H. J. (1992). Kleine Weltgeschichte der Philosophie. Frankfurt am Main: Fischer

Stumm, G., \& Voracek, M. (2009). Wörterbuch der Psychotherapie (2. Aufl.). Wien: Springer.

Tamir, D. I., \& Mitchell, J.P. (2012). Disclosing information about the self is intrinsically rewarding. Proceedings of the National Academy of Sciences, 109(21), 8038-8043.

Wirtz, M. A. (Hrsg.). (2014). Dorsch - Lexikon der Psychologie. Bern: Hogrefe.

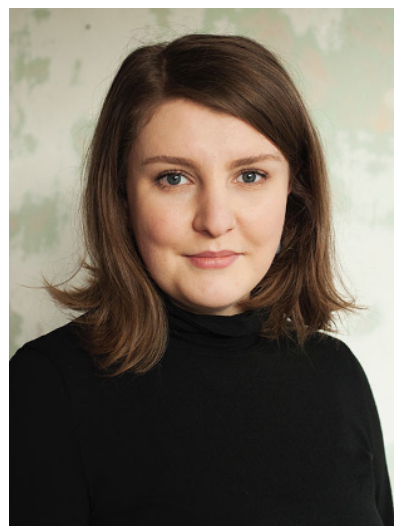

Milena Albiez studierte an der Kunsthochschule Kassel Visuelle Kommunikation (M.F.A) und an der Universität Kassel Mehrdimensionale Organisationsberatung, Supervision und Coaching (M. A.). Sie arbeitet als künstlerische Mitarbeiterin an der Kunsthochschule Kassel und ist deutschlandweit als Supervisorin, Coach und Organisationsberaterin tätig. Sie ist ifag ${ }^{\left({ }^{(}-\right.}$ zertifizierte Gruppendynamikerin und DGSv-zertifizierte Beraterin. www.milenaalbiez.de 